\title{
Considerando el Enfoque del Diseño de Microinteracciones como Aspecto Clave en el Desarrollo de Software
}

\author{
Jesús Hernández, Mónica A. Carreño, J. Andrés Sandoval, Italia Estrada \\ Universidad Autónoma de Baja California Sur \\ $\{$ jhernandez, mcarreno, sandoval, iestrada\}@uabcs.mx
}

\begin{abstract}
Resumen. La búsqueda de la perfección en el diseño de nuevas tecnologías ha impulsado la concepción de nuevos enfoques y entender los distintos y variados fenómenos envueltos en este proceso evolutivo. Vivimos en un mundo donde los mínimos detalles, las pequeñas cosas y los breves instantes son ya considerados realmente relevantes; esta aseveración se ha formado a partir de los descubrimientos consolidados al ahondar a mayores niveles de profundidad en la interacción entre personas y la tecnología digital. Es aquí donde aparecen con gran importancia las microinteracciones, pequeñas interacciones construidas como bloques moleculares en las interfaces, dando forma y consistencia a la trascendencia en el uso de una aplicación de software. Este trabajo busca establecer una postura conformando una serie de fundamentos significativos para aceptar a las microinteracciones como parte sustantiva en el desarrollo de software.
\end{abstract}

Palabras clave: Diseño de interacción, microinteracciones, interfaces, usuarios, aplicaciones.

\section{Introducción}

La expansiva cobertura de las distintas tecnologías en las actividades humanas nos han obligado a reflexionar sobre su impacto y trascendencia. Probablemente, porque no hay más remedio: las nuevas tecnologías ya forman parte de las costumbres, hábitos, cultura y relaciones sociales. Están aquí y no parece que vayan a desaparecer [1]. Esto ha conducido en el transcurso de las últimas dos décadas, la suma de nuevos y mayores esfuerzos para propiciar una mejor convivencia entre los dispositivos tecnológicos y sus principales usuarios, los seres humanos.

En consecuencia, esto también ha impactado a las aplicaciones de software, al menos es lo que se espera de ellas, pues hoy en día, un usuario común se ha convertido en un agente dinámico, capaz de desarrollar participaciones más activas basadas en el ejercicio de múltiples roles. El usuario promedio actual puede actuar, ya sea como el inconfundible operador de un sistema transaccional, o como un peculiar productor de nuevos contenidos [6]. La evolución y amplia variedad de aplicaciones le permiten transitar dentro de una pluralidad de roles, tales como operador, 
productor, cliente, participante, colaborador, miembro de una comunidad digital, entre muchos otros.

Esta transición ha sido favorecida en gran medida, por el Diseño Centrado en el Usuario (DCU), bajo la orientación del factor humano como eje central. Esta filosofía de diseño ha permitido el desarrollo y utilización de nuevos productos tecnológicos, incorporándolos a las actividades cotidianas de las personas, y a la vez propiciando de forma determinante su consideración como verdaderas herramientas de soporte y de contribución significativa [6].

El software no ha sido la excepción, las compañías desarrolladoras y programadores independientes han generado una gran oleada de aplicaciones bajo el mismo enfoque del DCU. Han entendido que el software debe idearse como un producto de calidad, útil y valioso, integrando características y funciones mediante una interfaz que deberá proyectar claramente su capacidad de cómo conseguir la satisfacción de las necesidades y deseos de los usuarios [7]. Una gran interfaz dispone en definitiva la balanza a favor de la aceptación y éxito de una aplicación.

A partir del contenido del presente artículo se pretende establecer una propuesta de enfoque a utilizar durante el diseño de aplicaciones, específicamente apuntalar al diseño de interacción como un requisito obligatorio para el diseño de mejores interfaces de usuario, muy cercanas al ser humano. Asimismo, se puede considerar como un llamado a la promoción de la relevancia de las microinteracciones, esas interacciones al detalle, que arman un tejido de pequeñas soluciones destinadas a construir mejores experiencias de uso para los individuos.

\section{Estado del arte}

Con los primeros estudios sobre usabilidad y psicología cognitiva realizados por Donald A. Norman, junto a la investigación de Ben Shneiderman sobre Interacción Hombre-Computadora (IHC) y los aportes del pionero Bill Moggridge dentro de la disciplina del Diseño de Interacción, han llevado a aceptar que la tecnología digital no sólo se trata de ofrecer alta tecnología, llena de sofisticadas funciones de gran desempeño, sino que además deben conjugar una disposición de interfaces y usos, en las formas más convenientes para el ser humano.

Derivados de tales estudios e investigaciones surgieron otros fundamentos y disciplinas que resultaron convenientes en el entendimiento de la interacción. Estas interrelaciones están presentadas a través de la figura 1, la cual muestra con claridad la estrecha relación entre estas múltiples disciplinas, y señala la inclusión de varios fundamentos identificados como significativos en un proceso de diseño con enfoque incluyente.

Es necesario señalar que la misma figura aborda con delimitación lo relacionado con el desarrollo de software, por lo que fue adaptada de su versión original para buscar una mejor explicación, y no confundir con la inclusión de otras disciplinas más relacionadas con el diseño de otro tipo de productos. 


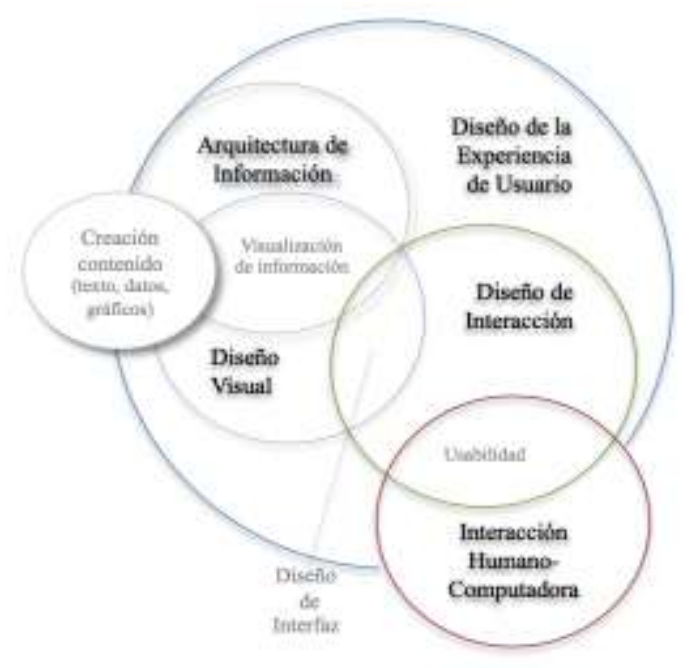

Figura 1. Representación de la relación del Diseño de Interacción con otras disciplinas. Creada a partir de la figura presentada por Saffer [8].

A partir de la figura 1, se derivan las siguientes definiciones como pertinentes:

El Diseño Centrado en el Usuario (DCU), es una filosofía de diseño que coloca al usuario de un producto, aplicación, o actividad (experiencia), como el centro de mayor atención en el proceso de diseño [6].

El Diseño de Interacción (DI), es todo lo concerniente a nuestra vida junto a la tecnología interactiva, como computadoras, telecomunicaciones, teléfonos móviles, entre otros [2]. El DI define la estructura y el comportamiento de los sistemas interactivos, donde los diseñadores de interacción se esfuerzan por crear lazos significativos entre las personas, productos y servicios que utilizan, desde computadoras, dispositivos móviles, aparatos y otros productos que van aún más allá [3].

Las macrointeracciones están relacionadas con la forma en que los usuarios interactúan con la interfaz, con la intención de ejecutar las operaciones claves que les lleven a culminar las tareas significativas [8]. Es posible considerar al conjunto de prestaciones o serie de funciones principales presentes a lo largo y ancho de la interfaz de una aplicación. Con las macrointeracciones se busca cumplir con los propósitos buscados en el trabajo de los usuarios.

Como una microinteracción, se considera a ese momento relativo a un producto o dispositivo que gira en torno a un caso de uso en particular - una pequeña parte de la funcionalidad que únicamente realiza una acción individual [4]. A través de las microinteracciones se llevan a cabo los detalles interactivos y funcionales de un producto, dispositivo o aplicación de software.

Existe una relación de pertenencia entre las macro y las microinteracciones (Fig. 2). Una macrointeracción puede integrar una o varias microinteracciones, y estas a su vez, pueden contener si es necesario, otras microinteracciones. 


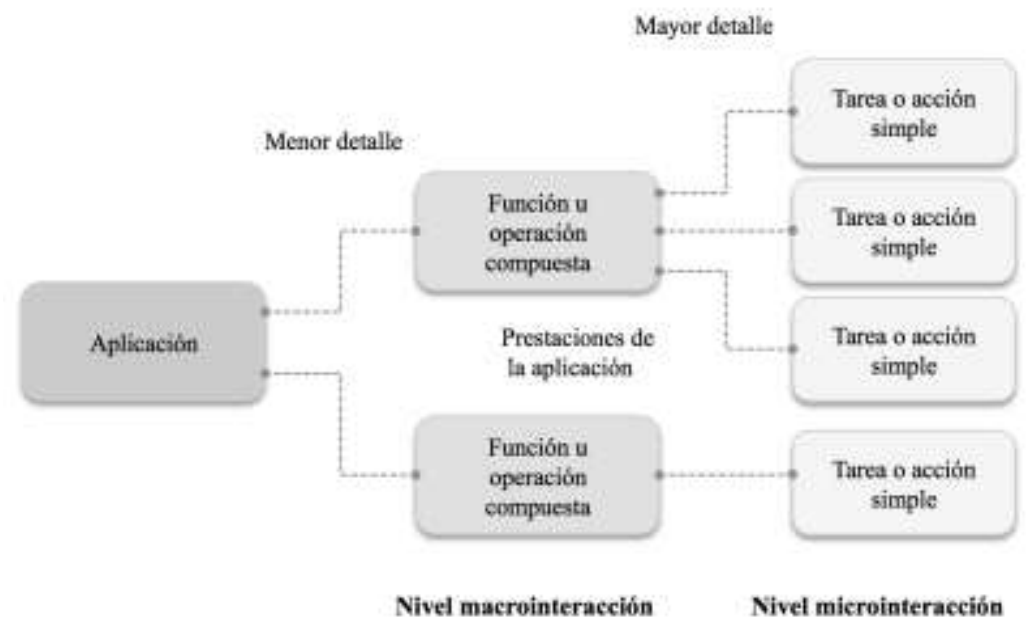

Figura 2. Representación de los niveles de interacción de una aplicación de software.

La especificación se detalla de izquierda a derecha, en el extremo izquierdo aparece la interacción desde el nivel de aplicación, en este punto se encuentra una visión general de la interacción del sistema. Hacia el lado derecho, se muestran las macrointeracciones y microinteracciones, donde el nivel de detalle aumenta conforme el diagrama se extiende horizontalmente en ese mismo sentido.

\section{Relevancia y Estructura de las Microinteracciones}

Con la conceptualización anterior es posible conducir la atención en la microinteracciones. A pesar de que pueden pasar desapercibidas por los usuarios por su mínimo tamaño o casi invisibilidad, las microinteracciones son muy importantes, ya que pueden ser útiles para:

- Ejecutar una tarea simple.

- Interconectar dispositivos.

- Interactuar con datos simples o básicos.

- Controlar algún proceso en ejecución.

- Ajustar un parámetro de configuración.

- Visualizar o crear una pequeña pieza de contenido como un mensaje de estado.

- Iniciar, detener o cambiar alguna función.

En una aplicación las microinteracciones son frecuentemente las responsables de convertirla en un producto predilecto o en otros casos, simplemente en algo tolerado. $\mathrm{Su}$ trascendencia puede ir aún más allá, pueden hacer la vida de las personas más sencilla, divertida e inclusive más interesante, si son bien construidas. Las microinteracciones se conforman bajo la siguiente estructura de elementos (Fig. 3): 


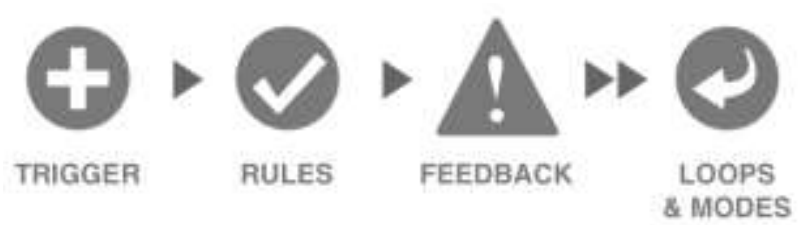

Fig. 3. Elementos que conforman la estructura de una microinteracción [4].

De acuerdo a la figura 3, los elementos de una microinteracción son:

- Un iniciador (trigger) es cualquier cosa que inicia o dispara una microinteracción. Los iniciadores manuales son disparados bajo el control del usuario, y puede ser un icono, una voz, un sonido, un toque, o comando gestual. Los iniciadores automáticos son manejados por el sistema bajo ciertas condiciones y reglas de ejecución bien definidas.

- Las reglas (rules) definen un modelo no técnico de la microinteracción, estableciendo lo que se puede hacer y en que orden. Las reglas deben reflejar y manejar restricciones de negocio, condicionamientos contextuales y técnicos. Es importante aprovechar lo que se sabe del usuario, plataforma, o el entorno para mejorar la microinteración.

- La retroalimentación (feedback) consiste en el entendimiento de lo que el usuario necesita conocer con oportunidad. En adición, son también importantes para entender las reglas de la microinteracción, identificando cuales de ellas merecen retroalimentación.

- Los ciclos \& modos (loops \& modes), se debe considerar los modos o modalidades de acción en correspondencia a la posible frecuencia de utilización, así como la distinción de la situación en curso para realizar las reacciones inmediatas y correspondientes. Los ciclos se refieren a las adaptaciones de las microinteracciones para extender su vida, requieren parámetros para asegurar la mejor experiencia del usuario. Los ciclos largos permiten crear un historial, útil para develar o cancelar aspectos a través del tiempo.

\section{Consideraciones en el Proceso de Diseño}

Un producto de software debe ser fácil de usar, accesible, flexible y de eficiente interacción. Todas estas características serán la fórmula perfecta para la interacción persona-computadora [5]. Por ello, el proceso de diseño de interacción debe ser abordado adecuadamente, incluyendo distintos niveles de interacción.

Por lo general, las microinteracciones son las últimas partes en ser diseñadas y desarrolladas, y por lo tanto, son frecuentemente ignoradas. Pero pasarlas por alto puede significar un error. Si se desarrollan con carencias, las funciones principales, sin importar que tan agradables sean, estarán envueltas por sufrimiento y frustración [4]. 
El diseño de una aplicación es tan bueno como lo sea en sus componentes más pequeños, en muchos de los casos los mínimos detalles desatan grandes emociones. Para los humanos la emoción es lo que dirige las cosas y las microinteracciones pueden estar ligadas a ellas. A continuación de describen algunos aspectos generales a considerar para el proceso de diseño de elementos macro y microinteractivos.

\subsection{Diseño de macrointeracciones}

En el nivel del macrodiseño de interacción de una interfaz, es necesario obtener un marco de referencia determinante para sentar las bases para el desarrollo del diseño a niveles más detallados y específicos (microdiseño). La intención de trabajo tiene que ir con una visión top-down, es decir, proceder con un enfoque de arriba hacia abajo.

Para este tipo de diseño resultan convenientes las herramientas del tipo wireframes y storyboards, consideradas como esenciales para mostrar y describir secuencias de pantallas, así como cuadros de diálogos que son llevados a cabo por los usuarios cuando ejecutan sus labores [9]. Como resultado debe mostrarse como se puede acceder a la funcionalidad de las tareas individuales.

El diseño de macrointeracciones de una interfaz de usuario tiene que ver con el panorama ofrecido por el cuadro grande de la interacción.

\subsection{Diseño de microinteracciones}

En el diseño de este nivel es preciso establecer claramente el entendimiento entre macrointeracción y microinteracción, debido a que algunos miembros de un equipo de desarrollo pueden llegar a confundirse o pensar que es lo mismo.

Para el diseño de microinteracciones hay que tener siempre en mente lo siguiente:

- Identificar el contexto,

- Pretender ser tan humano como el usuario lo es,

- Reciclar lo existente,

- No generalizar,

- Alejarse de la arbitrariedad e

- Inyectar emociones positivas a las interacciones.

Debido a la implicación de detalles dinámicos de los componentes individuales de la interfaz, es necesario usar diferentes recursos para diseñar su interacción. Realizar amplias descripciones textuales o storyboards con amplio detalle narrativo, son recursos muy valiosos; sin embargo, pueden aparecer algunas pequeñas interacciones que sean muy complicadas de definir con alguna herramienta o recurso disponible, por lo que quedará a la experiencia y habilidades del equipo de desarrollo para transmitir o comunicar lo que se desea conseguir como sensación de interacción [9].

Una vez lograda una definición y ajuste de expectativas, se puede pasar a la elaboración de prototipos desde dos perspectivas posibles [9]:

El diseño de interacción por componente. La modalidad de perspectiva de diseño de interacción por componente, consiste en definir las microinteracciones por 
componente, es decir, cada componente se trata con independencia. No se busca ninguna relación ni alineación de comportamientos individuales para conformar un comportamiento mayor.

El diseño de interacción concertada. Esta perspectiva también considera al comportamiento de los componentes o controles individuales, pero a la vez busca alinear o sincronizar aquellos que se comportan de forma semejante. Para esto, se identifican dimensiones claves para ubicar los componentes similares, y así definirlos con semejanza. Una configuración de parámetros centrales puede ser útil en esta idea.

En resumen, como aspectos principales a considerar serían: mantener siempre la conciencia del contexto -lo cual es fundamental para el diseño de microinteracciones-, la información de los usuarios, la plataforma presente y el entorno existente. Disponer de toda esta información y aprovecharla con el sentido de predecir acciones que pueden llegar a convertirse en una apreciación placentera para los usuarios por funcionalidad y apariencia estética, se puede valorar como un gran diferencial.

\section{Conclusiones}

El trabajo de desarrollo de software es mayormente concebido a macro escala, en frecuentes ocasiones los detalles son ignorados, o bien son turnados a un espacio de tiempo posterior, como la fase de pruebas o mantenimiento.

El diseño de interacción debe ser considerado principalmente durante las etapas de análisis de requerimientos y diseño de software, y vivirá hasta la etapa de pruebas para validar resultados y de ser necesario recibirá retroalimentación que podrá utilizar para redefinir aspectos de la interfaz de usuario durante el tiempo que sea necesario para su mejora paulatina.

Lo anterior, no resulta en nada una labor sencilla, conlleva la satisfacción de todo un espectro de requerimientos identificados para una aplicación. Por ello, involucra la aplicación de conocimientos y experiencias de buen nivel por los desarrolladores de software, pues la actualidad exige soluciones informáticas oportunas y concretas a problemas y necesidades de muchas formas y dimensiones, que en muchas ocasiones son difíciles de abordar.

Afortunadamente, han surgido nuevos paradigmas y enfoques, como propuestas para distinguir los mejores caminos de abordaje, siendo vital atenderlos para idear su incorporación de los nuevos productos de software. Aunado a esta divergencia, el factor humano ha trascendido con mayor exigencia de interacciones adecuadas a través de vivencias agradables y espléndidas en el uso de aplicaciones de cualquier tipo.

Como reciente dinámica, las microinteracciones han aparecido como una promesa al aseguramiento de una grata experiencia de usuario, en base a un correcto diseño pueden impulsar hacia la distinción entre una buena interfaz de usuario y una de gran trascendencia.

En consecuencia, el diseño a nivel de microinteracciones está tomando mayor importancia y a la vez adquiriendo un papel esencial respecto a la experiencia de usuario. Esto debe tomarse como un indicador a considerar para todo profesional independiente o equipo de desarrollo de software. Asimismo, es prioritario 
responder ágilmente a los planteamientos novedosos de enfoques que apoyen la redefinición de estrategias y estilos envueltos en todo el proceso de desarrollo de software. Resulta también importante, aplicar la misma incorporación de forma seria y permanente a todos los proyectos de software producidos en el contexto educativo, a través de las distintas asignaturas relacionadas con la programación.

Finalmente, hay que entender que las interfaces de usuario como producto final de participación interdisciplinaria, significan por ende logros estéticos para su aceptación, pero además también significan elevar la productividad de colaboradores, aceptación de clientes, afianzamiento con proveedores, y en general, el incremento de la aceptación del ser humano.

\section{Referencias}

1. Ogalde Carreaga, I., González Videgaray, M.: Nuevas tecnologías y educación. Diseño, desarrollo, uso y evaluación de materiales didácticos. Trillas, México (2009)

2. Moggridge, B.: Designing interactions. MIT Press, 1 ra. edición (2006)

3. Interaction Design Association, http://www.ixda.org/about/ixda-mission. Consultado el 10 de Junio de 2014.

4. Saffer, D.: Microinteractions. O'Reilly Media; 1ra. edición (2013)

5. Hernández, J., Carreño, M., Sandoval, J., Estrada, I., Aispuro F., Suárez, J.: Aplicación Móvil como Asistente en la Comunicación de Personas Discapacitadas y con Limitaciones del Habla. En: Memorias del 6to. Congreso Internacional en Ciencias Computacionales, pp. 30-35. Facultad de Ciencias, UABC, (2013)

6. Pratt, A.: Interactive Design: An Introduction to the Theory and Application of Usercentered Design. Rockport Publishers (2012)

7. Pannafino, J.: Interdisciplinary Interaction Design: A Visual Guide to Basic Theories, Models and Ideas for Thinking and Designing for Interactive Web Design and Digital Device Experiences. Assiduous Publishing, 1ra. edición (2012)

8. Saffer, D.: Designing for Interaction: Creating Innovative Applications and Devices. New Riders, 2da. edición (2009)

9. User Interface Architectures, http://www.centigrade.de/en/article/micro-interactions-vsmacro-interactions. Consultado el 19 de Junio de 2014. 